\title{
An Introduction to Electrodynamics and Special Relativity
}

\author{
Bernard J. Feldman \\ University of Missouri-St. Louis, St. Louis, USA
}

\begin{abstract}
This paper describes a paradox in electromagnetic theory involving a charge moving with a velocity $v$ parallel to a current carrying wire. In the frame of reference of the wire, the charge feels a magnetic force. In the frame of reference of the charge, there is no magnetic force, but there is an electric force due to the wire becoming charged. The charge on the wire can only be explained by length contractions from the theory of special relativity. This paradox is resolved both qualitatively and quantitatively.
\end{abstract}

Keywords: electrodynamics, special relativity, Lorentz equation, Gauss's Law, Ampere's Law

\section{Introduction}

Unfortunately, most introductory courses in electromagnetism do not mention special relativity, even though electromagnetism screams for special relativity. It was the contradictions in electromagnetism that motivated Albert Einstein to develop the theory of special relativity. This paper will present a simple paradox involving the Lorentz equation $\mathrm{F}=\mathrm{q}(\mathrm{E}+v \mathrm{x} \mathrm{B})$ that is an easy way to introduce special relativity into electrodynamics in high school and college introductory physics courses.

The paradox is the following: Consider a positive charge q moving in the $+x$-direction with a constant velocity $v$. Next to the charge is a wire carrying a positive current I also in the $+x$-direction (see Figure 1a). By Gauss's Law, the current creates no electric field E, because the wire is electrically neutral but by Ampere's Law, it does create a magnetic field $\mathrm{B}$ coming out of the paper at the moving charge. From the Lorentz equation with $\mathrm{E}=0, \mathrm{~F}=\mathrm{q} v \times \mathrm{B}$, there is a force on the charge towards the wire, causing the charge to accelerate towards the wire. Now consider a different frame of reference that is at rest with respect to the moving charge (see Figure 1b). In this frame of reference, the velocity of the charge is 0 , so the Lorentz force on the charge q, F', is zero, and charge is not accelerating towards the wire. Does the charge accelerate towards the wire or does it not?

Bernard J. Feldman, professor, Department of Physics and Astronomy, University of Missouri-St. Louis. 


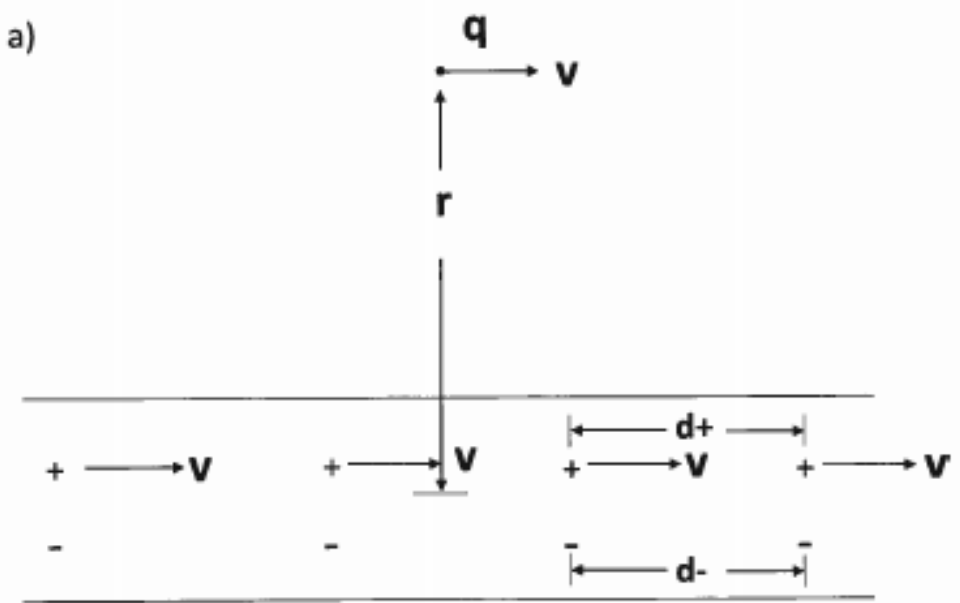

b)

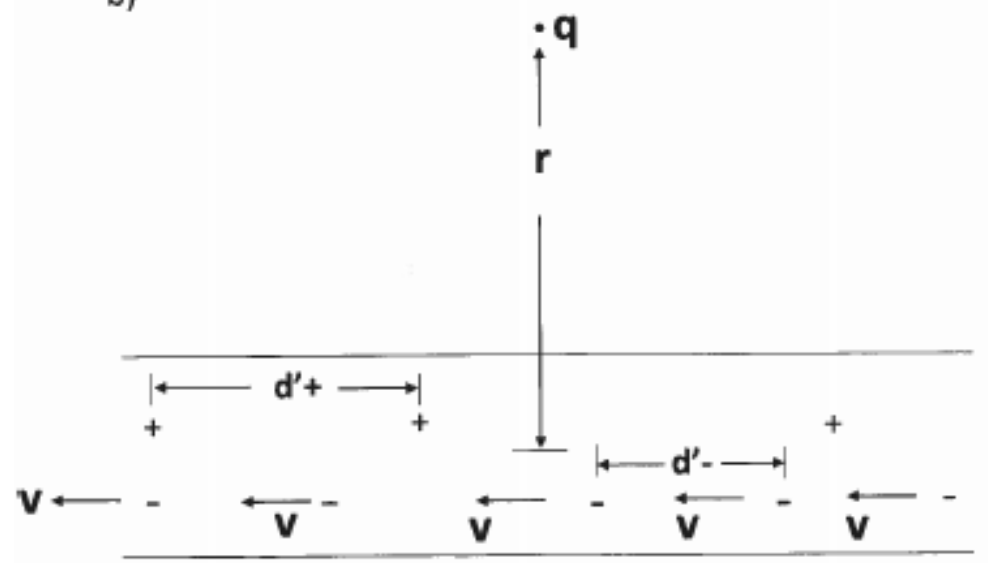

Figure 1. (a) First frame of reference. Sketch of charge q moving at velocity $v$ and a wire carrying a current of positive ions moving also at a velocity $v$; (b) Second frame of reference. Sketch of a charge at rest and a wire carrying a current of negative ions moving at a velocity, $-v$.

\section{Qualitative Solution}

Let me first give you the qualitative solution to this paradox. In the first frame of reference, think of the current $\mathrm{I}$ as consisting of a line of positive charges moving at a velocity $v$ in the $+x$ direction and a line of negative charges at rest. The distance between each positive charge is $d+$ and the distance between each negative charge is $d-$. Since this current carrying wire is electrically neutral, $d+=d-$.

Now, think of $\mathrm{d}+$ and $\mathrm{d}-$ as little meter sticks, $\mathrm{d}+$ moving at a velocity $v$ and $\mathrm{d}-$ at rest. Let us transform $\mathrm{d}+$ and $\mathrm{d}$ - to the second frame of reference where $\mathrm{q}$ is at rest. In this second frame of reference, the line of positive charges is at rest and separated by $\mathrm{d}^{\prime}+$ and the line of negative charges is moving at velocity, $v$, in the minus $x$-direction and separated by d'- (see Figures $1 \mathrm{a} \& 1 \mathrm{~b}$ ). From special relativity, we know that when we transform a meter stick from one frame of reference to another, a moving meter stick is shorter than the same meter stick at rest - the Lorentz contraction. In the first frame of reference, $\mathrm{d}+$ is a moving meter stick and $\mathrm{d}-$, a stationary one. In the second frame of reference, $\mathrm{d}$ ' + is longer than $\mathrm{d}+$, because it is stationary and d'- is shorter than $\mathrm{d}-$, because it is moving. That means in the second frame of reference, the density of positive charge in the 
wire is decreased and the density of negative charge is increased. Consequently, the wire is negatively charged, and that negative charge exerts an attractive force on the stationary positive charge, q. Now, in both frames of reference, q feels a force towards the wire, and the paradox is resolved.

\section{Quantitative Solution}

Now, let us redo the same problem in a quantitative way, following the derivation of Richard Feynman (Feynman, Leighton, \& Sands, 1964). In the first frame of reference, the current $\mathrm{I}=\mathrm{e} n_{0} \mathrm{~A} v$, where e is the charge of the moving and stationary charges in the wire, $n_{0}$ is the density of positive and negative charges in the wire, A is the cross-sectional area of the wire, and $v$ is the velocity of the positive charges in the wire. By Ampere's Law, the magnetic field at the moving charge, $\mathrm{q}, \mathrm{B}=\mu_{0} \mathrm{I} / 2 \pi r$, where $\mu_{\mathrm{o}}$ is a constant, the permeability of free space, and $r$ is the perpendicular distance from $\mathrm{q}$ to the wire. Finally, the force on $\mathrm{q}$ is given by the Lorentz equation (see Appendix),

$$
\mathrm{F}=\mathrm{q}(\mathrm{E}+v \times \mathrm{B})=\mathrm{q} v\left(\mu_{0} \mathrm{e} n_{0} \mathrm{~A} v / 2 \pi \mathrm{r}\right)
$$

where $\mathrm{E}=0$ because the wire is electrically neutral.

In the second frame of reference, the density of stationary positive charges in the wire, $n_{+}$, is reduced by the Lorentz factor, because $\mathrm{d}+$ has been increased to d'+ by the Lorentz factor. Thus, $n_{+}=n_{0}\left(1-v^{2} / c^{2}\right)^{1 / 2}$. Also, the density of negative charges moving at velocities $-v, n_{-}$, is increased by the Lorentz factor, because $\mathrm{d}$ - has been decreased to d'- by the Lorentz factor. Thus, $n_{-}=n_{0}\left(1-v^{2} / c^{2}\right)^{-1 / 2}$, where $\mathrm{c}$ is the speed of light. Now, the wire has a net negative charge density,

$$
n_{-}-n_{+}=n_{0}\left[\left(1-v^{2} / c^{2}\right)^{-1 / 2}-\left(1-v^{2} / c^{2}\right)^{1 / 2}\right]=n_{0}\left(1-v^{2} / c^{2}\right)^{-1 / 2}\left[1-\left(1-v^{2} / c^{2}\right)\right]=n_{0}\left(1-v^{2} / c^{2}\right)^{-1 / 2}\left(v^{2} / c^{2}\right)
$$

From Gauss' Law, the electric field at the charge $\mathrm{q}$ is $\mathrm{E}=\lambda / 2 \pi \varepsilon_{0} \mathrm{r}$, where $\lambda$ is the net charge per unit length, and $\varepsilon_{0}$ is a constant, the permittivity of free space. $\lambda=\mathrm{e}\left(n_{-}-n_{+}\right) \mathrm{A}$.

Plugging into the Lorentz equation,

$$
\mathrm{F}^{\prime}=\mathrm{q}\left(\mathrm{E}^{\prime}\right)=\mathrm{qe}\left(n_{-}-n_{+}\right) \mathrm{A} / 2 \pi \varepsilon_{0} \mathrm{r}=\mathrm{qe} n_{0}\left(1-v^{2} / c^{2}\right)^{-1 / 2}\left(v^{2} / c^{2}\right) \mathrm{A} / 2 \pi \varepsilon_{0} \mathrm{r}
$$

Using the fact that $c^{2}=1 / \varepsilon_{0} \mu_{0}$,

$$
\mathrm{F}^{\prime}=\mathrm{F}\left(1-v^{2} / c^{2}\right)^{-1 / 2}
$$

This is the correct relativistic transformation of a force perpendicular to $v$. In the limit of $v$ much less than $c, \mathrm{~F}^{\prime}=\mathrm{F}$. The paradox is quantitatively resolved.

\section{Conclusions}

Clearly, the above derivation where both $\mathrm{q}$ and the positive charges in the wire have the same velocity is a special case. The more general case where $\mathrm{q}$ and the positive charges in the wire have different velocities has been worked out by Purcell (1985) and is included in the Appendix; the ideas behind this more general derivation are the same as the simpler derivation above, but the mathematics is much more complicated.

Let me make some final comments. First, notice that the charge q creates an electric and magnetic field in the first frame of reference but only an electric field in the second frame of reference. And, the current creates only a magnetic field in the first frame of reference, but both an electric and magnetic field in the second frame 
of reference. It becomes clear that charges create both electric and magnetic fields (electromagnetic fields), and electric and magnetic fields in one frame of reference transform into different electric and magnetic fields in a different frame of reference.

Second, notice that the magnitude of the force is different in different frames of reference. That is because the force is not a four-component vector, and it is only the magnitude of four-vectors that are the same in all inertial frames of reference. An elementary discussion of this can be found in Feldman (2015). In contrast to F, the perpendicular distance from $q$ to the wire, $r$, does not change in different frames of reference; from special relativity, only distances parallel to $v$ are contracted or expanded. Notice that $\mathrm{q}$ and $\mathrm{e}$ are the same in both frames of reference; this is a result of experimental observations, not any theoretical basis (Feynman et al., 1964).

Third, at first glance, it appears that the wire is not charged in the first frame of reference, but is charged in the second. Actually, the wire is neutral in both frames of reference. Remember that a current is always a loop and whatever net charge is on one side of the loop nearest $\mathrm{q}$, the opposite charge is on the other side of the loop, because the current is going in the opposite direction.

Finally, notice one can start with the fact that $\mathrm{F}^{\prime}=\mathrm{F}$ at velocities much less than the speed of light and derive $c^{2}=1 / \varepsilon_{0} \mu_{0}$. Of course, one can get to the same result from Maxwell's equations, but that is a derivation beyond an introductory physics course.

\section{References}

Feldman, B. J. (2015). Why acceleration? US-China Educational Review A, 5(3), 223-228.

Feynman, R. P., Leighton, R. B, \& Sands, M. (1964). The Feynman lectures on physics, mainly electromagnetism and matter (pp. 13-6 \&13-9). Reading, Massachusetts: Addison-Wesley.

Purcell, E. M. (1985). Electricity and magnetism (2nd ed., p. 192). New York: McGraw-Hill. 


\section{Appendix}

Following the derivation of Edward M. Purcell (1985), let us consider the more general case where $\mathrm{q}$ is moving at $v_{0}$, different than $\mathrm{v}$, the velocity of the positive charges in the wire. Now, in the first frame of reference, the Lorentz force on $\mathrm{q}$ is

$$
\mathrm{F}=\mathrm{q} v_{0}\left(\mu_{0} \mathrm{e} n_{0} \mathrm{~A} v / 2 \pi \mathrm{r}\right)
$$

In the second frame of reference where the velocity of $q$ is 0 , the velocity of the positive charges in the wire must be first calculated using the relativistic formula for velocity addition.

$$
v^{\prime}=\left(v-v_{0}\right) /\left(1-v v_{0} / \mathrm{c}^{2}\right)
$$

Next, the Lorentz factor for $v^{\prime}$ must be calculated

$$
\begin{gathered}
\left(1-v^{, 2} / c^{2}\right)^{-1 / 2}=\left[1-\left(v-v_{0}\right)^{2} / \mathrm{c}^{2}\left(1-v v_{0} / \mathrm{c}^{2}\right)^{2}\right]^{-1 / 2}=\left(\mathrm{c}^{2}-v v_{0}\right)\left(\mathrm{c}^{4}-\mathrm{c}^{2} v^{2}-\mathrm{c}^{2} v_{0}^{2}+v^{2} v_{0}^{2}\right)^{-1 / 2} \\
=\left(1-v v_{0} / \mathrm{c}^{2}\right)\left(1-v_{0}^{2} / \mathrm{c}^{2}\right)^{-1 / 2}\left(1-v^{2} / \mathrm{c}^{2}\right)^{-1 / 2}
\end{gathered}
$$

using a considerable amount of algebra.

The density of negative charge in the wire $n_{-}$is increased by the Lorentz factor.

$$
n_{-}=n_{0}\left(1-v_{0}^{2} / c^{2}\right)^{-1 / 2}
$$

The density of the positive charge in the wire must first be decreased by calculating it in the frame of reference where the positive charge is at rest.

$$
n_{+}=n_{0}\left(1-v^{2} / \mathrm{c}^{2}\right)^{1 / 2}
$$

Then $\mathrm{n}_{+}$' must be increased by transforming it into the second frame of reference using eq. 7 .

$$
n_{+}=n_{+}\left(1-v v_{0} / \mathrm{c}^{2}\right)\left(1-v_{0}^{2} / \mathrm{c}^{2}\right)^{-1 / 2}\left(1-v^{2} / \mathrm{c}^{2}\right)^{-1 / 2}=n_{0}\left(1-v v_{0} / \mathrm{c}^{2}\right)\left(1-v_{0}^{2} / \mathrm{c}^{2}\right)^{-1 / 2}
$$

The net negative charge density on the wire is then

$$
n_{-}-n_{+}=n_{0}\left[\left(1-v_{0}^{2} / \mathrm{c}^{2}\right)^{-1 / 2}-\left(1-v v_{0} / \mathrm{c}^{2}\right)\left(1-v_{0}^{2} / \mathrm{c}^{2}\right)^{-1 / 2}\right]=n_{0}\left(v v_{0} / \mathrm{c}^{2}\right)\left(1-v_{0}^{2} / \mathrm{c}^{2}\right)^{-1 / 2}
$$

Finally, the force on $\mathrm{q}$ is

$$
\mathrm{F}^{\prime}=\mathrm{q}\left(\mathrm{E}^{\prime}\right)=\mathrm{qe}\left(n_{-}-n_{+}\right) \mathrm{A} / 2 \pi \varepsilon_{0} \mathrm{r}=\mathrm{qe} n_{0}\left(v v_{0} / \mathrm{c}^{2}\right)\left(1-v_{0}{ }^{2} / \mathrm{c}^{2}\right)^{-1 / 2} \mathrm{~A} / 2 \pi \varepsilon_{0} \mathrm{r}=\mathrm{F}\left(1-v_{0}{ }^{2} / \mathrm{c}^{2}\right)^{-1 / 2}
$$

which is identical to eq. 4. 